\title{
Microsurgical management of pediatric ependymomas of the fourth ventricle via the trans-cerebellomedullary fissure approach: A review of 26 cases
}

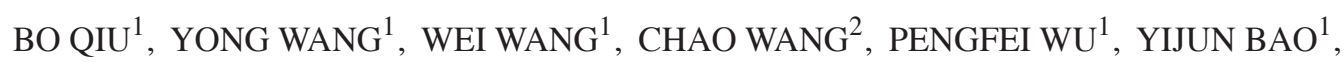 \\ SHAOWU OU ${ }^{1}$, ZONGZE GUO ${ }^{1}$ and YUNJIE WANG ${ }^{1}$ \\ ${ }^{1}$ Department of Neurosurgery, The First Affiliated Hospital of China Medical University, Shenyang, Liaoning 110001; \\ ${ }^{2}$ Department of Neurosurgery, The Affiliated Hospital of Medical College, Qingdao University, \\ Qingdao, Shandong 266003, P.R. China
}

Received March 8, 2015; Accepted April 1, 2016

DOI: $10.3892 / \mathrm{ol} .2016 .4507$

\begin{abstract}
In the present study, the microsurgical management of 26 ependymomas of the fourth ventricle in children via the trans-cerebellomedullary fissure (CMF) approach was reviewed and evaluated. Clinical data were obtained from 26 ependymomas of the fourth ventricle treated with microsurgery using the trans-CMF approach from March 2006 to September 2010 at the Department of Neurosurgery of The First Affiliated Hospital of China Medical University (Shenyang, China). These data were collected and analyzed. Suboccipital median posterior fossa craniotomy and trans-CMF approach were performed in all cases for the microsurgical removal of the tumors. An additional incision was performed in the inferior medullary velum of 5 patients, in order to obtain adequate exposure of the tumors. As a result, all tumors were well exposed during surgery. Gross total resection (GTR) was achieved in 22 cases, near total resection (NTR) in 3 cases and subtotal resection (STR) in 1 case. All excised tumors were pathologically confirmed. No mortality occurred intraoperatively, and no patient presented with mutism or any other surgery-related complications. One patient suffered from postoperative hydrocephalus and received ventriculoperitoneal shunting, which relieved the symptoms. Over the 3.0-7.5-year follow-up period (mean, 4.8 years), tumor relapse occurred in 1 case with GTR, 2 cases with NTR and 1 case with STR. In total, 3 patients succumbed to tumor relapse and 4 were lost to follow-up. According to the literature and the clinical experience of the present authors, the trans-CMF approach
\end{abstract}

Correspondence to: Professor Bo Qiu, Department of Neurosurgery, The First Affiliated Hospital of China Medical University, 155 North Nanjing Street, Shenyang, Liaoning 110001, P.R. China

E-mail:bo_qiu@msn.com

Key words: cerebellomedullary fissure, fourth ventricle, ependymoma, microsurgery provides safe and sufficient access to the fourth ventricle without the requirement of an incision in the inferior vermis. This approach prevents damage to the normal cerebellum and improves the surgical outcome. Tumor removal, restoration of cerebrospinal fluid circulation and preservation of brainstem function are factors that should be taken into consideration during surgery. For patients with residual tumors, adjuvant radiotherapy and/or chemotherapy may be beneficial.

\section{Introduction}

Ependymomas may occur in any part of the ventricular system, but they mainly develop in the posterior fossa (1-3). The occurrence of ependymomas is more common in pediatric patients than in adult patients, accounting for $8-13 \%$ of all intracranial tumors in children and up to $4 \%$ of brain tumors in adults. Approximately one-half of ependymomas are found in children aged $<3$ years (1-13). In children, ependymomas usually occur in the fourth ventricle and display a series of symptoms and signs, including increased intracranial pressure, ataxia, vertigo, chronic cerebellar tonsillar hernia and brainstem dysfunction $(1,14)$. Despite the fact that infratentorial tumors exhibit a lower biological activity than supratentorial tumors (9,15-17), tumors of the posterior fossa carry a higher surgical risk, due to the technical difficulties in performing a complete excision, which may lead to treatment failure $(9,15)$.

Ependymomas of the fourth ventricle are usually approached with a posterior median craniotomy/craniectomy, followed by splitting of the vermis; however, this trans-vermian approach could easily injure the vermis, cerebellar dentate and globose nuclei, which may result in tremor, ataxia, atonia and cerebellar mutism, thereby affecting the prognosis $(13,14,18-25)$. The establishment of the novel trans-cerebellomedullary fissure (CMF) approach, which provides sufficient access to the fourth ventricle without sacrificing the inferior vermis, has been associated with favorable outcomes $(13,14,18-20,22-32)$. The trans-CMF approach is gaining popularity among the available surgical approaches that are used for the complete removal of fourth ventricular tumors excessively extending laterally into the cerebellomedullary cistern $(13,14,18,19,23,27,31,32)$. In 
children, ependymomas of the fourth ventricle usually originate from the ependymal layer and are well-circumscribed $(1,2)$. Also, ependymomas in the present study usually exhibted a cleavage plane between the tumor tissue and brainstem during the surgery, particularly in low-grade disease. Therefore, sufficient exposure and complete removal of the tumor may improve the prognosis to a great extent. The present study reports 26 consecutive cases of ependymomas of the fourth ventricle in children who received posterior fossa craniotomy via the trans-CMF approach for the excision of the tumors, and presents a review of the surgical procedures and findings.

\section{Patients and methods}

Patients. Between March 2006 and September 2010, 26 patients with ependymomas of the fourth ventricle underwent surgery at the Department of Neurosurgery of The First Affiliated Hospital of China Medical University (Shenyang, China). The clinical records of these cases were retrospectively reviewed, and the demographics and major clinical manifestations of the patients are listed in Table I. The patient population was composed of 15 male and 11 female pediatric patients (male:female ratio, 1.36:1) aged 2.5-14.0 years (mean age, 8.7 years). The clinical course of the patients lasted between 2 weeks and 3 years (median time period, 7.5 months). The most common symptoms included headache, dizziness, nausea and/or vomiting, cranial nerve deficits, brainstem dysfunction and hydrocephalus (Table I). A total of 3 patients exhibited obvious signs of hydrocephalus, including supratentorial ventricle enlargement, papilledema, urinary incontinence, unsteady gait and drowsiness. Of these 3 patients, 2 had received preliminary ventriculoperitoneal shunting in local hospitals in the month prior to surgery. The procedures in the present study were performed according to the ethical standards of the Declaration of Helsinki (33), and written informed consent was obtained from the legal guardian of each patient.

Neuroimaging studies. Contrast-enhanced magnetic resonance imaging (MRI; Signa HDxt 3.0T; GE Healthcare, Chalfont, UK) and/or computed tomography (CT; Brilliance 64-slice CT; Philips Medical Systems, Cleveland, OH, USA) scans were evaluated preoperatively in all cases. Imaging was used to evaluate the tumor size, anatomical boundaries, association with and compression of the brainstem, as well as extent of tumor invasion. The maximum tumor diameter range was 2.7-8.1 cm, with a mean diameter of $4.1 \mathrm{~cm}$. Supratentorial ventricular enlargement was present in 12 cases (46.2\%), and cerebellar tonsillar hernia and/or syringomyelia in 7 cases (26.9\%). Remarkable brainstem compression and displacement were observed in 14 cases $(53.8 \%)$. All ependymomas were observed to originate from the fourth ventricle, with slight and heterogeneous enhancement and occasional cystic degeneration, calcification and/or tumor apoplexy. Tumor invasion into the cisterna magna was observed in 7 cases $(26.9 \%)$, and corpora quadrigemina involvement in 1 case $(3.8 \%)$.

Surgical procedures. Following admission, all patients were routinely treated with $20 \%$ mannitol solution (China Otsuka Pharmaceutical Co., Ltd, Tianjin, China) to decrease intracranial pressure. To sufficiently relieve intracranial hypertension in the 12 patients with supratentorial ventricular enlargement, 3 patients with hydrocephalus received frontal ventriculostomy 2 or 3 days prior to tumor removal, and the other 9 patients underwent occipital ventriculostomy intraoperatively, prior to dural incision.

As previously described $(13,14,18-20,23,30)$, all patients were operated on in the prone position with the head flexed at the craniocervical junction. A vertical midline incision was performed, followed by a suboccipital craniotomy/craniectomy, with a bone window of $\sim 5.0 \times 4.0 \mathrm{~cm}$. If the lesion was unilateral, the craniotomy/craniectomy had to be extended to the contralateral side just across the midline. A $\sim 1.5-2.0-\mathrm{cm}$ wide section of the rim of the foremen magnum was removed, and then the posterior arch of the atlas was removed $(\sim 1.5 \mathrm{~cm}$ in width) if the tonsils or lesion extended distally to the spinal canal. The dura was opened with a Y-shaped incision. Following the identification of the foramen of Magendie and the posterior inferior cerebellar artery (PICA), the tonsils were dissected from the vermis and the CMF was opened. The CMF is composed of two slit spaces, the uvulotonsillar and medullotonsillar spaces $(14,19,23,26,27,32)$. These two spaces were sharply dissected to release the tonsils and the uvula, which enabled the easy retraction of the uvula superiorly and of the tonsils laterally, thereby exposing the inferior roof of the fourth ventricle, which is composed of the tela choroidea, inferior medullary velum and lateral recess $(14,18,24)$. Caution is required when dividing the attachments between the tonsil, fourth ventricular floor and inferior medullary velum. When the CMF was further opened, the exposure extended from the foramen of Magendie to the foramen of Luschka and four lower cranial nerves in the cerebellomedullary angle, depending on the tumor location and extension. In fact, less dissection of these structures was required in cases of large tumors, due to the distension at the foramen of Magendie produced by the tumor (Fig. 1). If the lesions were primarily located in the lower $3 / 4$ region of the ventricle, only the tela choroidea was opened; however, for tumors invading the upper $1 / 4$ area of the ventricle, both the tela choroidea and inferior medullary velum were opened to gain satisfactory exposure. When the tonsil was gently elevated, the tumor bulk was exposed and then dissected from the surrounding cerebellar and brainstem structures using microsurgical techniques. The suboccipital bone flaps were replaced in patients who did not present with brain swelling during surgery. All ependymomas were pathologically confirmed using hematoxylin and eosin staining and immunohistochemistry. Briefly, all excised tumors were formalin (Sigma-Aldrich, St. Louis, MO, USA)-fixed, paraffin (Sigma-Aldrich)-embedded and cut into 4- $\mu \mathrm{m}$ sections followed by deparaffinization. The sections were stained with hematoxylin and eosin (Beyotime Institute of Biotechnology, Haimen, China) and reviewed by two experienced neuropathologists. Immunohistochemical staining was performed using a Universal Dako LSAB2/HRP Kit (Dako, Glostrup, Denmark) according to the manufacturer's instructions, with 3,3'-diaminobenzidine (DAB; Dako) as a chromogen. Selected antibodies, including glial fibrillary acidic protein (GFAP; monoclonal mouse anti-human; 1:100; cat. no. M0761; Dako), epithelial membrane antigen (EMA; monoclonal mouse anti-human; 1:100; cat. no. M0613; Dako), S-100 (polyclonal rabbit anti-human; 1:1,000; cat. no. Z0311; Dako), vimentin 
(monoclonal mouse anti-human; 1:100; cat. no. M0725; Dako), Ki-67 (monoclonal mouse anti-Human; 1:50; cat. no. M7240; Dako), cytokeratin (monoclonal mouse anti-human; 1:100; cat. no. M0821; Dako), synaptophysin (monoclonal mouse anti-human; 1:50; cat. no. M7315; Dako), D2-40 (Podoplanin; monoclonal mouse anti-human; 1:100; cat. no. M3619; Dako) were used for antigen detection. All staining was visualized using an optical microscope (BX40; Olympus Corporation, Tokyo, Japan).

Extent of resection (EOR). The extent of resection was divided into three categories: Gross-total resection (GTR), near-total resection (NTR) and subtotal resection (STR). NTR was defined as a resection following which only residual tumor $<5$-mm thick was visible on postoperative neuroimaging. STR was defined as resection that left behind residual tumor $>5-\mathrm{mm}$ thick on postoperative neuroimaging. GTR was defined as a resection following which the tumor cells that remained were not visible under the operating microscope (Leica OH3 FL800; Leica Microsystems GmbH, Wetzlar, Germany); patients who achieved GTR did not display any evidence of disease on postoperative contrast-enhanced MRI. NTR was defined as a resection following which only $5-\mathrm{mm}$ thick residual tumor was visible on postoperative neuroimaging. STR was defined as a resection following which 5-mm thick residual tumor was visible on postoperative neuroimaging (8).

Postoperative care and follow-ups. Postoperative external ventricular drainage was not routinely used, since the normal cerebrospinal fluid (CSF) circulation was mandatorily restored in all patients during surgery. The patients received continuous intensive monitoring for respiratory and cardiovascular complications, and were symptomatically treated following surgery. In cases exhibiting shortness of breath and expectoration, tracheostomy was often required. For patients with residual tumors, postoperative radiotherapy was recommended, which was usually at a dose of 45-54 Gy administered in 30 fractions, with 5 fractions per week over 6 weeks. Patients were followed-up for 3, 6 and 12 months postoperatively, and annually thereafter. Follow-up CT and/or MRI scans were performed for the evaluation of the EOR and surgical strategy. Telephone interviews with patients and their families were also conducted.

\section{Results}

Among the 26 patients, simple tela choroidea opening was performed in 21 patients, while the other 5 patients received both tela choroidea and inferior medullary velum opening due to the excessive tumor invasion into the upper ventricle. Unilateral and bilateral approaches were performed in 17 and 9 cases, respectively. All ependymomas were pathologically confirmed. Of the 26 ependymomas, 19 were low-grade and 7 high-grade. No intraoperative mortality was recorded. One patient underwent permanent ventriculoperitoneal shunting for persistent hydrocephalus. Each ependymoma was well exposed during surgery, and GTR was achieved in 22 cases, NTR in 3 and STR in 1. Two patients who underwent the bilateral trans-CMF approach developed temporary mutism, with the symptom disappearing within 4 weeks. Other complications included ataxia, weakness in the extremities, mild facial or abducent palsy and dysphagia, which steadily improved and eventually recovered within 1 month following symptomatic treatment. No surgery-induced permanent neurological deficit was recorded. One patient succumbed to pulmonary infection 3 months subsequent to surgery. Over the 3.0-7.5-year follow-up period (average, 4.8 years), the preoperative symptoms disappeared in 20 cases while persisted in 1 . Tumor relapse occurred in 1 case with GTR, 2 cases with NTR and 1 case with STR. In total, 3 patients succumbed to tumor relapse and 4 were lost to follow-up.

\section{Discussion}

The fourth ventricle is situated in front of the cerebellum and behind the pons and upper half of the medulla oblongata, and it consists of several vital structures in the ventricle wall and floor, thus being a surgically challenging area due to the severe deficits that may occur if these delicate structures are injured $(9,14,19,32,34)$. Ependymoma is one of the most common tumors of the fourth ventricle, particularly in pediatric patients $(1,2,9)$. Due to its deep location and close association with the surrounding brain stem, important peripheral nerves and blood vessels, the radical surgical removal of ependymomas of the fourth ventricle is considerably risky $(9,14,19,30,34,35)$. Since pediatric patients are constantly growing and developing, their clinical manifestations lack specificity and accuracy in description (35). This feature was also observed in the present cases. Early manifestations of nervous system injuries are usually ignored, as noted in the current study, which is why tumors are generally large at diagnosis (35). In the present study, increased intracranial pressure and obstructive hydrocephalus were the most common early symptoms of ependymomas of the fourth ventricle. Due to the advances in MRI technology, it is possible to detect the precise location and extension of an ependymoma of the fourth ventricle $(2,36,37)$. Once a diagnosis of ependymoma of the fourth ventricle is confirmed, surgical resection of the tumor is usually obligatory due to oncological reasons, and must be as extensive as possible, in order to reduce local recurrence and seeding rates of metastatic cells $(5,6,17,38-40)$.

The traditional approach for removing ependymomas of the fourth ventricle is incision of the cerebellar vermis, which, however, is often associated with a higher incidence of cerebellar mutism or posterior fossa syndrome, along with chronic neurocognitive sequelae, compared with the trans-CMF appr oach $(13,18-21,24,25,41-43)$. The trans-CMF approach to the fourth ventricle, which does not cause significant injury to the neural tissue, was first proposed by Matsushima et al (36), and is currently widely accepted $(8,13,14,18-20,22-26,29-32,36,43)$. As a natural cleft between the cerebellum and medulla oblongata, the CMF provides sufficient exposure of the fourth ventricle without causing neurological deficits, following incisions in the tela choroidea and inferior medullary velum $(14,23,27)$. Furthermore, the trans-CMF approach is able to achieve a greater angle of exposure and more capacious working space than the trans-vermian approach $(14,18,22)$. There are two types of trans-CMF approach: The simple tela choroidea opening approach and the telovelar approach, and the decision to use intraoperatively one or the other depends on the location of the upper pole of the tumor in the fourth 
ventricle (14). Of the 26 ependymomas of the fourth ventricle reviewed in the present study, 21 (80.8\%)were removed using a simple tela choroidea opening, while $5(19.2 \%)$ required an incision in the inferior medullary velum in order to gain satisfactory exposure of the upper ventricle. Matsushima et al (18) introduced three opening methods for the trans-CMF approach: Extensive (aqueduct type), lateral wall and lateral recess opening types. According to our experience in the present study, the simple tela choroidea opening approach is sufficient in the majority of cases. When the tela choroidea is completely opened and the tonsils and uvula are properly retracted, the lateral recess and at least the lower 3/4 parts of the fourth ventricle are adequately visualized (14). Caution should be taken in order to prevent excessive stretching of the tonsils, which could lead to the compression of the associated dentate nuclei and cerebellar peduncles $(25,27,32,44)$.

If an ependymoma is relatively large, it frequently protrudes through the ventricular outlets into the subarachnoid space, expanding the operating space (20), as indicated in Fig. 1. In fact, the larger the tumor is preoperatively, the more stretched and thinned the inferior medullary velum and tela choroidea tend to be, which enables easier and wider access to the tumor within the ventricle $(13,20)$. Furthermore, additional operating space in large ependymomas may be gained by central debulking; however, 5 ependymomas of those reviewed in the present study involved the upper part of the fourth ventricle (upper 1/4 region, generally), which rendered an incision in the inferior medullary velum necessary in order to obtain adequate exposure (31). It has been previously reported that tonsillar resection in the telovelar approach could provide a better operative perspective compared with tonsillar retraction (45); however, according to the experience of the present study, complete dissection of the tela choroidea and flexible retraction of the brain tissue are more important than tonsillar retraction.

Of the 26 cases of ependymoma of the fourth ventricle reviewed in the present study, 17 underwent the unilateral approach and 9 the bilateral approach. In general, following the dissection of the ipsilateral CMF and contralateral tonsillar retraction, the majority of tumors were sufficiently exposed, unless there was obvious invasion into the contralateral, superolateral and/or lateral recess. In the present study, extensive involvement of the fourth ventricle was observed in 9 cases, where the bilateral approach was performed; however, temporary postoperative mutism occurred in 2 patients $(7.7 \%)$, both of whom underwent the bilateral trans-CMF approach. According to the existing literature $(19,29)$, the bilateral trans-CMF approach appears to be associated with a high rate of postoperative mutism, compared with the unilateral approach. Despite the controversy surrounding the pathophysiological mechanisms, it is considered that the dentate nuclei and their connections with the thalamus through the brainstem participate in speech function; therefore injury to this pathway may result in mutism $(12,14,20,21)$. Another explanation for this observation may be that the preservation of the contralateral CMF reduces the likelihood of blood vessel injury (particularly in branches of the PICA) during the unilateral approach. Thus, the unilateral approach could be an effective way to avoid postoperative neurological deficits; however, the patient number in the present study was too small to draw any
Table I. Summary of patient characteristics from the 26 reviewed cases of ependymoma of the fourth ventricle.

\begin{tabular}{|c|c|}
\hline Characteristic & Value \\
\hline Patients, $\mathrm{n}$ & 26 \\
\hline \multicolumn{2}{|l|}{ Gender, $\mathrm{n}$} \\
\hline Male & 15 \\
\hline Female & 11 \\
\hline \multicolumn{2}{|l|}{ Disease course } \\
\hline Range & 2 weeks- 3 years \\
\hline Average & 7.5 months \\
\hline \multicolumn{2}{|l|}{ Age, years } \\
\hline Range & $2.5-14.0$ \\
\hline Mean & 8.7 \\
\hline \multicolumn{2}{|l|}{ Clinical features, n (\%) } \\
\hline Headache and/or dizziness & $15(57.7)$ \\
\hline Nausea and/or vomiting & $19(73.1)$ \\
\hline Cranial nerve deficits & $11(42.3)$ \\
\hline Abducent paralysis & $7(26.9)$ \\
\hline Dysphagia and/or hoarseness & $5(19.2)$ \\
\hline Tinnitus and/or deafness & $2(7.7)$ \\
\hline Trigeminal paralysis & $1(3.8)$ \\
\hline Ataxia, nystagmus and/or gait disturbance & $13(50.0)$ \\
\hline Motor and sensory dysfunction & $18(69.2)$ \\
\hline Simple vertigo & $4(15.4)$ \\
\hline Bruns sign & $3(11.5)$ \\
\hline Papilledema & $7(26.9)$ \\
\hline Consciousness disorder & $1(3.8)$ \\
\hline
\end{tabular}

conclusions. In the present study the bilateral approach was applied to large tumors, the resection of which was essentially more invasive than the unilateral approach, due to more anatomical structure being resected, leading to an increased chance of tissue injury. With regard to other complications, such as ataxia and cranial nerve and/or brainstem dysfunction, no pattern of unilateral or bilateral predominance was identified, which could be due to the small number of patients evaluated in the present study. Furthermore, the postoperative complications gradually improved and eventually disappeared, indicating the safety and usefulness of this minimally invasive approach.

Considering the particular nature and pathogenesis of ependymoma, the detailed microsurgical procedures performed for ependymomas of the fourth ventricle are relatively different from those performed for other tumor types (23). The extent of surgical resection is a major determinant of the outcome $(1,6,8,11,13,17,38-40)$. It is known that the majority of ependymomas arise from ependymal cells present in the floor of the fourth ventricle $(1,2,37)$. In the current study, the majority of tumors derived from or close to the obex, or close to the midline and located at the lower part of the ventricle (the midfloor type) $(2,13,22,37)$, which facilitated their exposure easier using the unilateral approach or limited dissection of the tela choroidea. Ependymomas are normally slow-growing, well-circumscribed, soft and often frond-like. In addition, they 
A

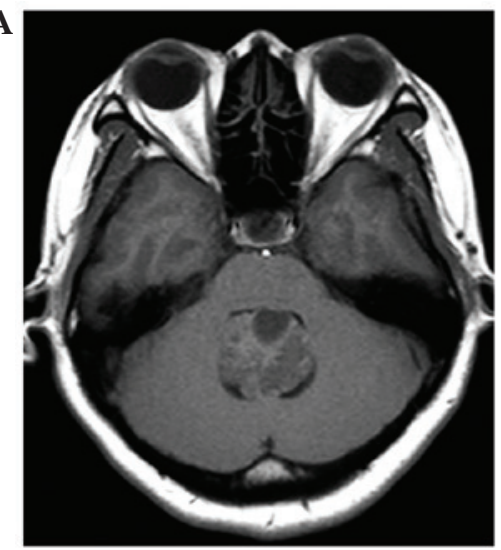

D

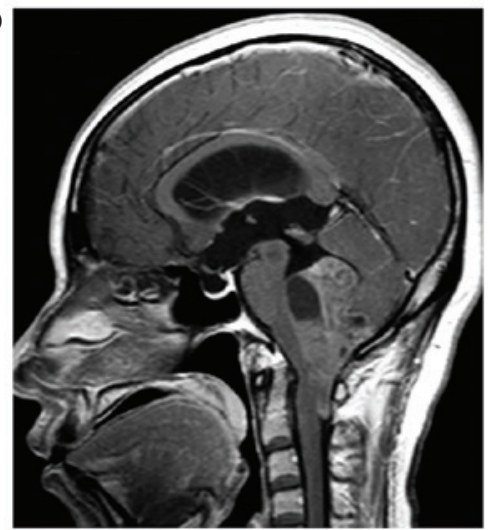

G

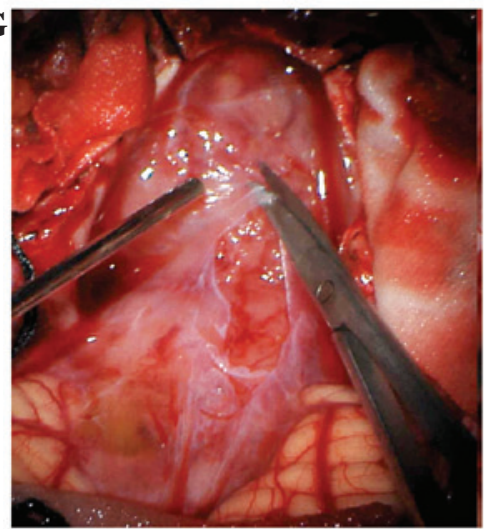

B

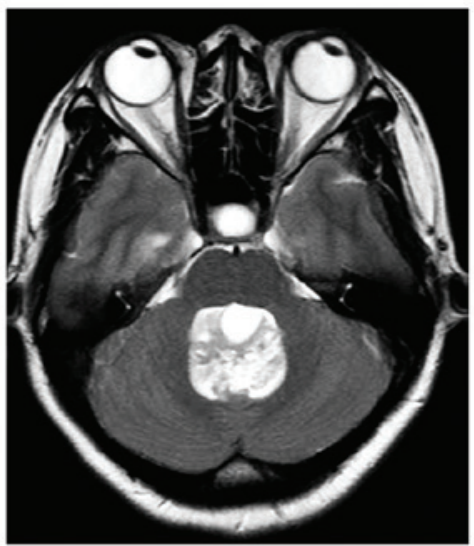

E
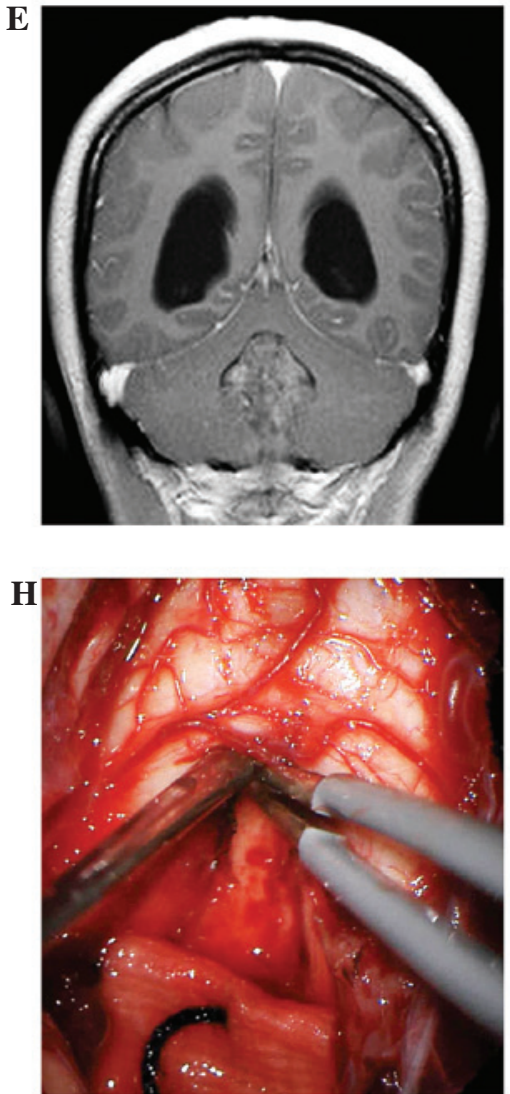
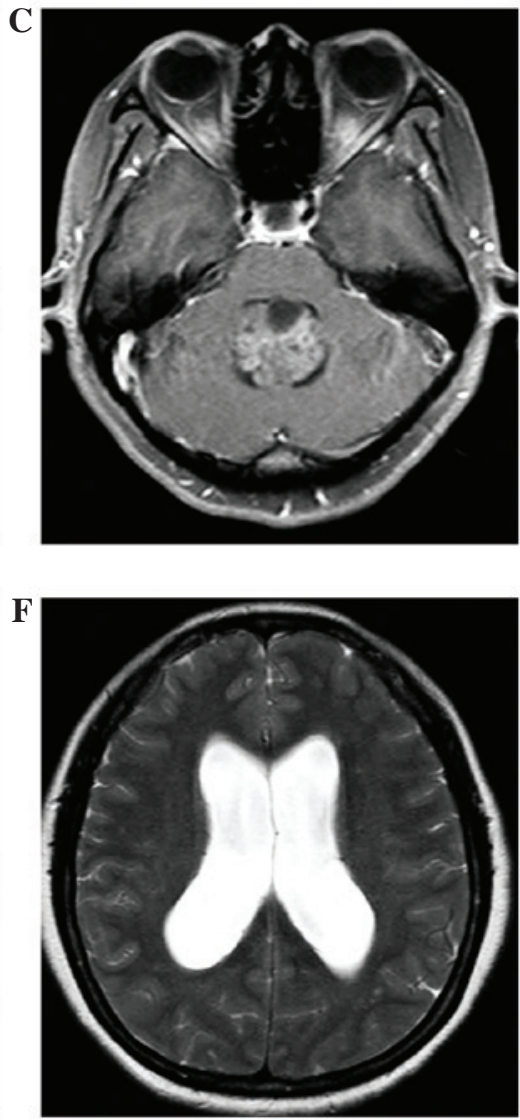

I

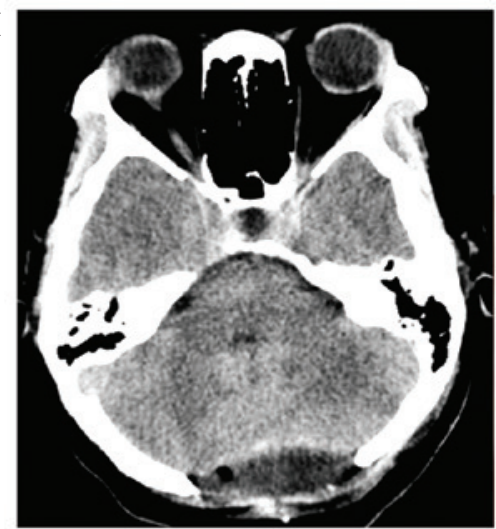

Figure 1. Illustration of a large ependymoma of the fourth ventricle with obstructive hydrocephalus. (A and B) Non-enhanced magnetic resonance imaging scans revealed (A) an iso- to slightly hypointense lesion in the fourth ventricle on T1-weighted images and (B) an iso- to hyperintense lesion in the fourth ventricle on T2-weighted images. (C) Axial, (D) sagittal and (E) coronal T1-weighted imaging showed the lesion was heterogeneously and mildly enhanced following gadolinium administration $(0.1 \mathrm{mmol} / \mathrm{kg}$; Omniscan; GE Healthcare). (F) A supratentorial hydrocephalus was observed, which was due to the obstruction of the fourth ventricle. $(\mathrm{G}$ and $\mathrm{H}$ ) Intraoperatively, distal extension of the tumor to the spinal canal was observed. Following the removal of the vast majority of the tumor in the spinal canal, cerebellomedullary cistern and fourth ventricle, the tumor was observed to originate from the obex, since the obex was obviously infiltrated. In consequence, a near total resection was performed. (I) A computed tomography scan performed 2 weeks post-surgery revealed that the tumor had been removed and the morphology of the fourth ventricle had become normal.

have a propensity for plastic growth and may protrude into the cerebellopontine cistern $(1,2)$. Therefore, ependymomas of the fourth ventricle may be well-exposed and easily recognized, following their separation from the surrounding structures and management of the tumor base. The well-delineated tumors exhibited distinct boundaries from the surrounding tissues, and the soft and heterogeneous nature of the parenchyma facilitated the tumor removal. Intraoperatively, the detachment of the tumor base is of particular importance, since any injury to the medulla oblongata could have serious consequences.
Conversely to astrocytomas, whose infiltrating nature hinders a precise estimation of tumor removal both intraoperatively and on postoperative imaging, the ependymomas reviewed in the present study (particularly the low-grade ependymomas), usually displayed a cleavage plane between tumor tissue and brainstem, which facilitated the tumor removal. Since the tumor was minimally adherent and could be easily dissected from the base, the EOR of the ependymomas improved, compared with other types of tumors of the fourth ventricle, including high-grade ependymomas. However, great care 
A
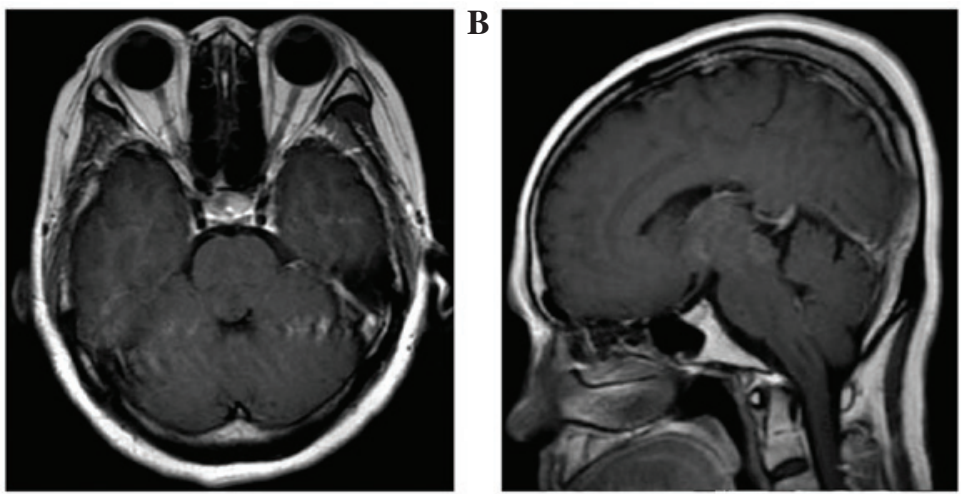

D

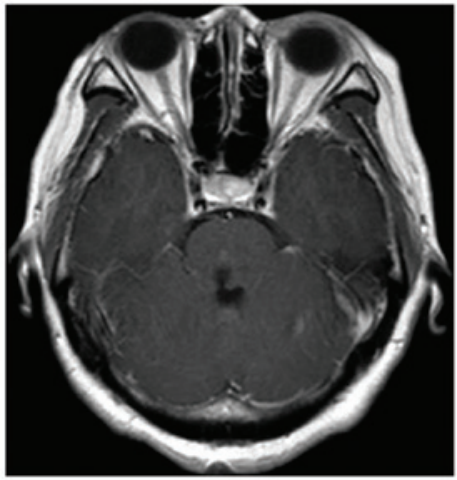

$\mathbf{E}$

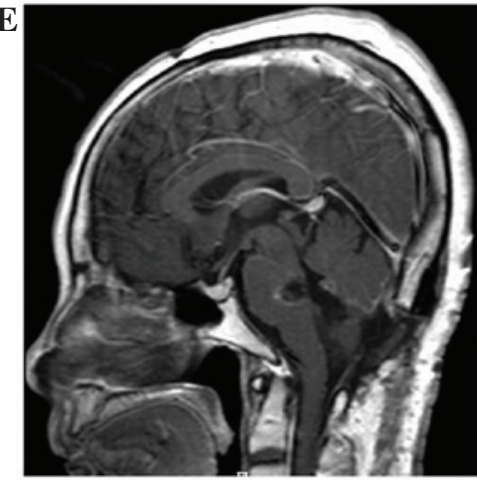

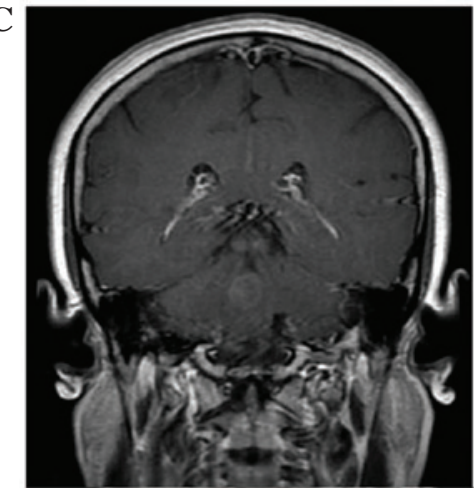

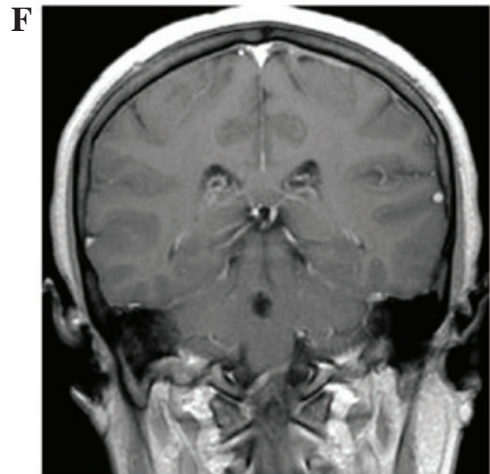

G
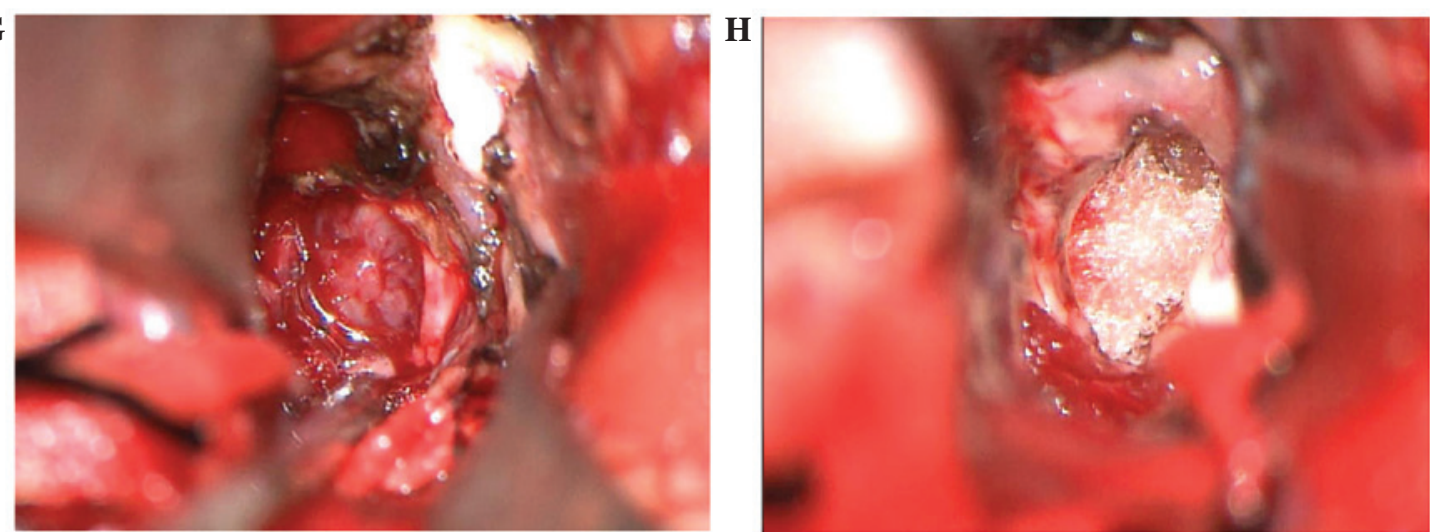

Figure 2. Illustration of an intrinsic ependymoma of the pons. Preoperative (A) axial, (B) sagittal and (C) coronal MRI scans revealed a scarcely enhanced tumor in the fourth ventricle located within the pons. The tumor was preoperatively diagnosed as an astrocytoma. (D) Axial, (E) sagittal and (F) coronal enhanced T1-weighted MRI scans performed 3 months following surgery indicated gross total resection of the tumor. (G) Intraoperatively, a longitudinal incision of the pons was performed, exposing a well-circumscribed tumor. (H) Following the excision of the tumor, the resection cavity was filled with absorbable hemostatic cotton and gelatin sponge (Johnson \& Johnson Medical, New Brunswick, NJ, USA). The final pathological diagnosis confirmed an ependymoma. MRI, magnetic resonance imaging.

should be taken when the tumor base is near the obex, which is pivotal to respiratory function (23). In one of the reviewed cases in the present study, the tumor had extensively invaded the obex, and was therefore managed aggressively. The patient suffered from respiratory and expectoration dysfunction, and eventually succumbed to pulmonary infection. Therefore, if the floor of the fourth ventricle, including the obex, is obviously infiltrated, then GTR should not be a mandatory goal, in order to avoid vital damage (Fig. 1). In addition, as represented in Fig. 2, one of the ependymomas reviewed in the present study was preoperatively diagnosed as an intrinsic brainstem tumor (low-grade astrocytoma), since it did not display typical imaging features of ependymoma and was located within the pons. Intraoperatively, the tumor was well demarcated, and was easily separated from the surrounding tissue following a longitudinal incision of the pons. The final pathological diagnosis confirmed a low-grade ependymoma.

In addition, several aspects should be taken into consideration in order to achieve a safe GTR: The branches of the PICA will be encountered during the dissection of the CMF, and these arteries should be carefully protected (with the exception of those well-defined tumor feeding arteries). If any damage occurs to the PICA branches distal to the medullary branches at the level of the roof of the fourth ventricle, inability to stand or walk and nystagmus without appendicular dysmetria may occur $(19,31)$. Furthermore, any reflux vein should be managed with great caution, particularly those near the obex. Intraoperatively, it is also important to ensure the patency of the fourth ventricle and 
aqueduct, thereby relieving the obstructive hydrocephalus. In all the cases included in the present study, smooth CSF circulation was visualized; however, a patient presented with postoperative hydrocephalus and underwent ventriculoperitoneal shunting. Postoperative hydrocephalus may be caused by brain tissue swelling, residual tumor or arachnoid adhesion due to incomplete bipolar coagulation hemostasis. Neurosurgeons should also note that ependymomas have a tendency to disseminate through the CSF, with CSF seeding present in 3-15\% of the cases $(6,11,12,28)$. Therefore, during surgery, the cisterna magna and aqueduct should be protected using brain cotton, in order to prevent tumor cells from spreading through the ventricular system.

In conclusion, the trans-CMF approach constitutes a favorable surgical approach for the treatment of ependymomas of the fourth ventricle, since it does not require the splitting of the vermis. The opening portion of the CMF and roof of the ventricle should be determined based on the location, extension and size of the lesion. For the majority of cases, the unilateral trans-CMF approach with a simple opening of the tela choroidea is sufficient and should be preferred, since it is minimally invasive and could potentially prevent the occurrence of postoperative mutism. The total removal of tumors focally attached to critical areas of the fourth ventricle should not be attempted at the expense of the patient's morbidity and mortality. Tumor removal, restoration of the CSF circulation and preservation of brainstem function should all be taken into consideration during surgery. If appropriate microneurosurgical techniques are used via the trans-CMF approach, the majority of ependymomas of the fourth ventricle may be accessed and resected safely.

\section{Acknowledgements}

The present study was supported by the Natural Science Foundation of Liaoning Province (Shenyang, China; grant no. 2013021075 awarded to Professor Bo Qiu), the Fund for Scientific Research of The First Affiliated Hospital of China Medical University (Shenyang, China; grant no. fsfh 1304 awarded to Professor Bo Qiu), the Program for Liaoning Excellent Talents in University (Shenyang, China; grant no. LJQ2013085 awarded to Professor Pengfei Wu), the Liaoning Provincial Project on Social Development (Shenyang, China; grant no. 2013225079 awarded to Professor Shaowu Ou) and the Science and Technology Program of Shenyang City (Shenyang, China; grant no. F12-277-1-04 awarded to Professor Shaowu Ou).

\section{References}

1. Spagnoli D, Tomei G, Ceccarelli G, Grimoldi N, Lanterna A, Bello L, Sinisi MM, De Santis A and Villani RM: Combined treatment of fourth ventricle ependymomas: Report of 26 cases. Surg Neurol 54: 19-26, 2000.

2. Tortori-Donati P, Fondelli MP, Cama A, Garrè ML, Rossi A and Andreussi L: Ependymomas of the posterior cranial fossa: CT and MRI findings. Neuroradiology 37: 238-243, 1995.

3. Kun LE, Kovnar EH and Sanford RA: Ependymomas in children. Pediatr Neurosci 14: 57-63, 1988.

4. Kojima A, Yamaguchi N, Okui S, Kamiya M, Hirato J and Nakazato Y: Parenchymal anaplastic ependymoma with intratumora hemorrhage: A case report. Brain Tumor Pathol 20: 85-88, 2003.

5. El Majdoub F, Elawady M, Blau T, et al: Intracranial ependymoma: Long-term results in a series of 21 patients treated with stereotactic (125)iodine brachytherapy. PLoS One 7: e47266, 2012.
6. Rudà R, Gilbert M and Soffietti R: Ependymomas of the adult: Molecular biology and treatment. Curr Opin Neurol 21: 754-761, 2008.

7. Nazar GB, Hoffman HJ, Becker LE, Jenkin D, Humphreys RP and Hendrick EB: Infratentorial ependymomas in childhood: Prognostic factors and treatment. J Neurosurg 72: 408-417, 1990.

8. Morris EB, Li C, Khan RB, Sanford RA, Boop F, Pinlac R, Xiong $X$ and Merchant TE: Evolution of neurological impairment in pediatric infratentorial ependymoma patients. J Neurooncol 94: 391-398, 2009.

9. Ferrante L, Mastronardi L, Schettini G, Lunardi P and Fortuna A: Fourth ventricle ependymomas. A study of 20 cases with survival analysis. Acta Neurochir (Wien) 131: 67-74, 1994.

10. Kilday JP, Rahman R, Dyer S, Ridley L, Lowe J, Coyle B and Grundy R: Pediatric ependymoma: Biological perspectives. Mol Cancer Res 7: 765-786, 2009.

11. Kano H, Yang HC, Kondziolka D, Niranjan A, Arai Y, Flickinger JC and Lunsford LD: Stereotactic radiosurgery for pediatric recurrent intracranial ependymomas. J Neurosurg Pediatr 6: 417-423, 2010.

12. Bademci G, Tun K, Erden E, Evliyaoglu C and Unlu A: Late dissemination of ependymoma: Case report. Neurocirugia (Astur) 18: 333-336, 2007.

13. Shimoji K, Miyajima M, Karagiozov K, Yatomi K, Matsushima T and Arai H: Surgical considerations in fourth ventricular ependymoma with the transcerebellomedullary fissure approach in focus. Childs Nerv Syst 25: 1221-1228, 2009.

14. Han S, Wang Z, Wang Y and Wu A: Transcerebellomedullary fissure approach to lesions of the fourth ventricle: Less is more? Acta Neurochir (Wien) 155: 1011-1016, 2013.

15. Goldwein JW, Leahy JM, Packer RJ, Sutton LN, Curran WJ, Rorke LB, Schut L, Littman PS and D'Angio GJ: Intracranial ependymomas in children. Int J Radiat Oncol Biol Phys 19: 1497-1502, 1990.

16. Goldwein JW, Glauser TA, Packer RJ, Finlay JL, Sutton LN, Curran WJ, Laehy JM, Rorke LB, Schut L and D'Angio GJ: Recurrent intracranial ependymomas in children. Survival, patterns of failure, and prognostic factors. Cancer 66: 557-563, 1990.

17. Guyotat J, Signorelli F, Desme S, Frappaz D, Madarassy G, Montange MF, Jouvet A and Bret P: Intracranial ependymomas in adult patients: Analyses of prognostic factors. J Neurooncol 60: 255-268, 2002.

18. Matsushima T, Abe H, Kawashima M and Inoue T: Exposure of the wide interior of the fourth ventricle without splitting the vermis: Importance of cutting procedures for the tela choroidea. Neurosurg Rev 35: 563-572, 2012.

19. Gök A, Alptekin M and Erkutlu I: Surgical approach to the fourth ventricle cavity through the cerebellomedullary fissure. Neurosurg Rev 27: 50-54, 2004.

20. Zaheer SN and Wood M: Experiences with the telovelar approach to fourth ventricular tumors in children. Pediatr Neurosurg 46: 340-343, 2010.

21. Puget S, Boddaert N, Viguier D, Kieffer V, Bulteau C, Garnett M, Callu D, Sainte-Rose C, Kalifa C, Dellatolas G and Grill J: Injuries to inferior vermis and dentate nuclei predict poor neurological and neuropsychological outcome in children with malignant posterior fossa tumors. Cancer 115: 1338-1347, 2009.

22. Ziyal IM, Sekhar LN and Salas E: Subtonsillar-transcerebellomedullary approach to lesions involving the fourth ventricle, the cerebellomedullary fissure and the lateral brainstem. Br J Neurosurg 13: 276-284, 1999.

23. El-Bahy K: Telovelar approach to the fourth ventricle: Operative findings and results in 16 cases. Acta Neurochir (Wien) 147: 137-142, 2005.

24. Kawashima M, Matsushima T, Nakahara Y, Takase Y, Masuoka J and Ohata K: Trans-cerebellomedullary fissure approach with special reference to lateral route. Neurosurg Rev 32: 457-464, 2009.

25. Akakin A,Peris-Celda M, Kilic T, Seker A, Gutierrez-Martin A and Rhoton A Jr: The dentate nucleus and its projection system in the human cerebellum: The dentate nucleus microsurgical anatomical study. Neurosurgery 74: 401-425, 2014.

26. Abla AA and Lawton MT: Cerebellomedullary fissure dissection and tonsillar mobilization: A gateway to lesions around the medulla. World Neurosurg 82: e591-e592, 2014.

27. Di Ieva A, Komatsu M, Komatsu F and Tschabitscher M: Endoscopic telovelar approach to the fourth ventricle: Anatomic study. Neurosurg Rev 35: 341-349, 2012.

28. Kawabata Y, Takahashi JA, Arakawa Y and Hashimoto N: Long-term outcome in patients harboring intracranial ependymoma. J Neurosurg 103: 31-37, 2005. 
29. Matsushima T, Inoue T, Inamura T, Natori Y, Ikezaki K and Fukui M: Transcerebellomedullary fissure approach with special reference to methods of dissecting the fissure. J Neurosurg 94: 257-264, 2001

30. Matsushima T, Kawashima M, Inoue K, Matsushima $\mathrm{K}$ and Miki K: Exposure of wide cerebellomedullary cisterns for vascular lesion surgeries in cerebellomedullary cisterns: Opening of unilateral cerebellomedullary fissures combined with lateral foramen magnum approach. World Neurosurg 82: e615-e621, 2014.

31. Mussi AC and Rhoton AL Jr: Telovelar approach to the fourth ventricle: Microsurgical anatomy. J Neurosurg 92: 812-823, 2000.

32. Rajesh BJ, Rao BR, Menon G, Abraham M, Easwer HV and Nair S: Telovelar approach: Technical issues for large fourth ventricle tumors. Childs Nerv Syst 23: 555-558, 2007.

33. World Medical Association: World Medical Association Declaration of Helsinki: Ethical principles for medical research involving human subjects. JAMA 310: 2191-2194, 2013.

34. Parkinson D: The posterior cranial fossa: Microsurgical anatomy and surgical approaches. Neurosurgery 48: 1196, 2001.

35. Chen D, Wei X, Yin Q, Guan J, Pan W, Wang C and Liu Y: The microscopic surgical treatment for tumor of posterior cranial fossa in children. Clin Oncol Cancer Res 6: 95-99, 2009.

36. Matsushima T, Fukui M, Inoue T, Natori Y, Baba T and Fujii K: Microsurgical and magnetic resonance imaging anatomy of the cerebello-medullary fissure and its application during fourth ventricle surgery. Neurosurgery 30: 325-330, 1992.

37. Ikezaki K, Matsushima T, Inoue T, Yokoyama N, Kaneko Y and Fukui M: Correlation of microanatomical localization with postoperative survival in posterior fossa ependymomas. Neurosurgery 32: 38-44, 1993.
38. Pollack IF, Gerszten PC, Martinez AJ, Lo KH, Shultz B, Albright AL, Janosky $J$ and Deutsch M: Intracranial ependymomas of childhood: Long-term outcome and prognostic factors. Neurosurgery 37: 655-667, 1995.

39. Robertson PL, Zeltzer PM, Boyett JM, Rorke LB, Allen JC, Geyer JR, Stanley P, Li H, Albright AL, McGuire-Cullen P, et al: Survival and prognostic factors following radiation therapy and chemotherapy for ependymomas in children: A report of the Children's Cancer Group. J Neurosurg 88: 695-703, 1998.

40. Sutton LN, Goldwein J, Perilongo G, Lang B, Schut L, Rorke L and Packer R: Prognostic factors in childhood ependymomas. Pediatr Neurosurg 16: 57-65, 1990.

41. Dailey AT, McKhann GM II and Berger MS: The pathophysiology of oral pharyngeal apraxia and mutism following posterior fossa tumor resection in children. J Neurosurg 83: 467-475, 1995.

42. Mastronardi L: Mutism and pseudobulbar symptoms after resection of posterior fossa tumors in children: Incidence and pathophysiology and transient cerebellar mutism after posterior fossa surgery in children. Neurosurgery 38: 1066, 1996.

43. Van Calenbergh F, Van de Laar A, Plets C, Goffin J and Casaer P: Transient cerebellar mutism after posterior fossa surgery in children. Neurosurgery 37: 894-898, 1995.

44. Fulton JF and Dow RS: The cerebellum: A summary of functional localization. Yale J Biol Med 10: 89-119, 1937.

45. Jittapiromsak P, Sabuncuoglu H, Deshmukh P, Spetzler RF and Preul MC: Accessing the recesses of the fourth ventricle: Comparison of tonsillar retraction and resection in the telovelar approach. Neurosurgery 66 (Suppl Operative 3): 30-40, 2010. 